\title{
Simple scheme for realizing fast light with low distortion in optical fibers
}

\author{
Sanghoon Chin, Luc Thévenaz \\ Ecole Polytechnique Fédérale de Lausanne, STI-NAM Station 11, CH-1015 Lausanne, Switzerland \\ Miguel Gonzalez-Herraez \\ Department of Electronics, University of Alcalá de Henares, E-28805 Madrid, Spain
}

Fast light experiments are particularly challenging and fascinating for the scientific community, since superluminal signal velocities can be achieved (however preserving Einstein's causality). These experiments require inducing a very large anomalous dispersion in the medium at the signal frequency. Sharp atomic absorptions and electromagnetically-induced absorption (EIA) have provided a mean to obtain this large anomalous dispersion. In fibers, the narrowband loss of stimulated Brillouin scattering has been used to create these conditions. All these methods for obtaining fast light, however, have the common drawback of making the pulse propagate in a spectral region of high absorption. To overcome this impairment, two methods have been devised: one is to propagate the pulse in a region slightly detuned from a gain line, where the group velocity change is negative; the other, more sophisticated approach is to make use of the large anomalous dispersion appearing between two gain peaks. These methods have been previously demonstrated in atomic vapours. In a previous paper [1] we reported the first experimental demonstration of pulse advancement with gain in optical fibers using stimulated Brillouin scattering (SBS). We tested the two methods described above to achieve gain-assisted fast light and demonstrated experimentally that the method based on the double Brillouin gain peak produces pulse advancement with lower distortion. However, the distortion achieved in these experiments was still far from ideal, and the underlying scientific challenge to produce fast light with negligible distortion remains intact. In this paper we propose a simple scheme for achieving fast light with low distortion, based on the unique and flexible properties of Brillouin amplification. We demonstrate experimentally that this scheme delivers better results than the previously reported ones, and hence might open new possibilities in fundamental research on fast light.

A gain doublet is naturally created by appending two segments with similar length of different single mode fibers as SBS gain medium. The fibers show a different core doping concentration, so that their Brillouin spectra are clearly distinct: the central frequency difference $\left(\mathrm{V}_{\mathrm{B} 1}-\mathrm{V}_{\mathrm{B} 2}\right)$ is $120 \mathrm{MHz}$ and is ideally stable, while they show nearly identical natural linewidth of $25 \mathrm{MHz}$. When the SBS process in these fibers is activated by a single-frequency pump beam, a double SBS gain resonance is obtained after successively propagating through the 2 fibers. When a pulse is spectrally placed in a median position between these two gain resonances, it experiences fast light. To adjust the delay/distortion characteristics of the medium, the pump laser was directly modulated by means of a noise generator to broaden its spectrum [2], resulting in a controlled variation of the refractive index as a consequence of the spectral change. Figure 1(a) shows the gain doublet after propagating through the fibers with respect to different spectral widths of the pump. Figure 1(b) shows measured temporal advancements of the probe signal as a function of the pump power, showing that this scheme may produce fast light with lower distortion than previously reported results.
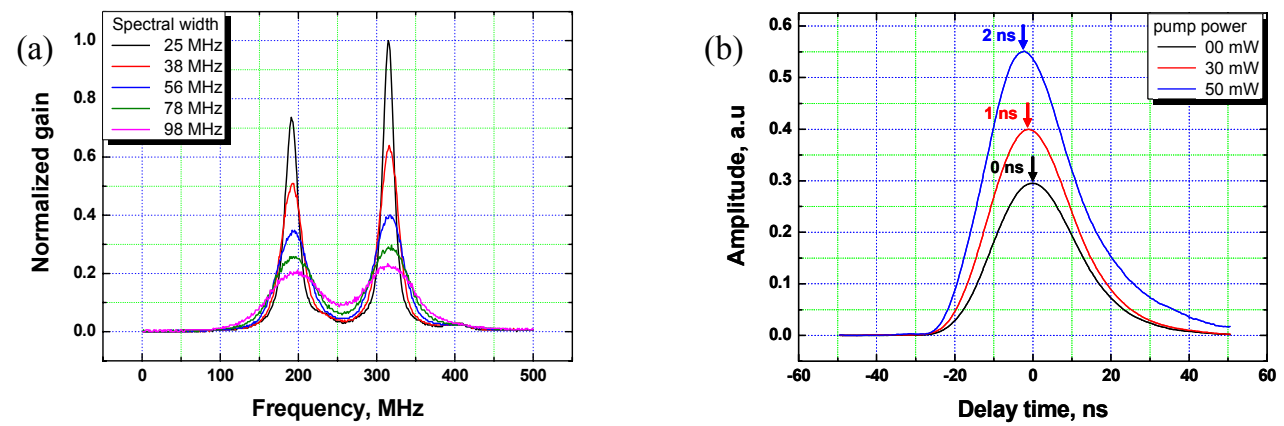

Fig.1. (a) Gain-doublet spectra as a function of frequency with respect to different bandwidths of the pump beam and (b) Measured pulse advancement with $38 \mathrm{MHz}$ pump spectral width, showing clearly low distortion on the pulses.

\section{Reference}

1. K. Y. Song, M. Gonzalez Herráez and Luc Thévenaz, Opt. Express 13, 9758-9765 (2005)

2. M. Gonzalez Herráez, K. Y. Song and Luc Thévenaz, Opt. Express 14, 1395-1400 (2005). 